\title{
Maximum Likelihood Estimation and Centroiding Hybrid RSSI-based Indoor Positioning
}

\author{
Liu Shan ${ }^{\mathrm{a}}$, Peng Shengliang ${ }^{\mathrm{b}}$, Wang Zhic \\ College of Information Science and Engineering, Huaqiao University, Xiamen, 361021, China

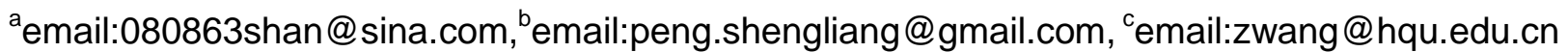

Keywords: Wireless positioning; RSSI; maximum likelihood estimation; centroiding algorithm

\begin{abstract}
RSSI-based positioning technology suffers from accuracy degradation due to complex indoor environment, and traditional weighted centroid algorithm hardly satisfies people's accuracy requirements. In this paper, a hybrid RSSI based positioning algorithm is proposed. Firstly, maximum likelihood estimation method is used to estimate the rough information of positioning target; then, optimized weighted centroiding algorithm is adopted to obtain its accurate coordinates, which further improves the positioning accuracy. Simulation results have verified the superiority of this hybrid algorithm compared with traditional algorithms.
\end{abstract}

\section{Introduction}

With the rapid development of wireless local area network technology in recent years, Location Based Services (LBS) have become more and more important. The outdoor positioning technique, such as Global Positioning System (GPS) [1], can provide accurate positioning and has been offering reliable navigation for transportation. However, the issue of indoor positioning still remains unconquered and attracts great attention. Based on whether measuring the distance between the nodes in positioning, current positioning algorithms are generally divided into two categories: range-based positioning and range-free positioning [2]. In detail, range-based positioning methods [3] include Received Signal Strength Indication (RSSI) based method [4], Time of Arrival based method, Time Difference of Arrival based method, and Angle of Arrival based method. Among them, the RSSI based method is distinguished by low cost and no need of extra hardware support, thus increasingly becomes a research hotspot.

Currently, various algorithms have been suggested for RSSI based positioning. Traditional centroiding positioning algorithm is simple and consumes little calculation, but its positioning accuracy is bad. Literature [5] combines RSSI and centroiding positioning algorithm by using RSSI as the weight of centroiding positioning, and hence proposes a weighted centroiding positioning algorithm. Literature ${ }^{0}$ suggests using the reciprocal of distance sum between unknown node and known node as the weighting factor. These two algorithms both improve the positioning accuracy, but their way to select the weight value is not optimum. Meanwhile, literature [6] advises to use maximum likelihood estimation to obtain the coordinates of unknown nodes. On the basis of literature [6] and literature [7], this paper proposes a hybrid positioning method by combining RSSI-based maximum likelihood estimation and weighted centroiding. The proposed method firstly acquires rough information of the position target through maximum likelihood estimation, and then takes into account different rough information in selecting the centroiding weight, which can effectively uplift positioning accuracy.

\section{Path Loss Model}

In wireless communications, signals will attenuate during propagation, and such attenuation is related to the transmission path. This is the key idea of RSSI based positioning. Frequently-used path loss models comprise free space transmission model, ground reflection (doublet) model, logarithm distance path loss model, logarithm-normal distribution model, and so on. In actual application, multipath and obstacles make the transmission model extremely complex [8]. So logarithm-normal distribution model is usually adopted as follows: 
$P(d)[d B]=P\left(d_{0}\right)+10 n \lg \left(\frac{d}{d_{0}}\right)+X_{\sigma}$

Where, $d$ refers to the distance between transmitter and receiver; $d_{0}$ refers to the near-ground reference distance; $P(d)$ refers to the signal loss at receiver through the propagation distance $d$; $P\left(d_{0}\right)$ refers to the signal loss of $d_{0}$ away from transmitter; $n$ refers to the environment-related path loss index, ranging from 2 to $6 ; X_{\sigma}$ follows zero mean and unit deviation Gaussian random distribution.

In actual scenarios, considering $d_{0}$ is one meter and $A$ is a Gaussian distribution random variable with the mean of -RSSI $(1 \mathrm{~m})$, the relationship between RSSI and $d$ is

$$
\operatorname{RSSI}(d)[d B]=-10 n \lg (d)-A
$$

\section{Maximum Likelihood Estimation}

The positioning principle of maximum likelihood estimation method is as follows: assuming in the position area there exist one unknown node to be positioned and $n$ known nodes, where the distances between unknown node and known nodes have been estimated according to the path loss model described above.

Considering the coordinates of the known nodes are $\left(x_{1}, y_{1}\right),\left(x_{2}, y_{2}\right),\left(x_{3}, y_{3}\right), \cdots,\left(x_{n}, y_{n}\right)$,the coordinate for the node to be positioned is $(x, y)$, and the estimated distances are $d_{1}, d_{2}, d_{3}, \cdots, d_{n}$, we can obtain the following equation set:

$$
\left\{\begin{array}{c}
\left(x_{1}-x\right)^{2}+\left(y_{1}-y\right)^{2}=d_{1}^{2} \\
\left(x_{2}-x\right)^{2}+\left(y_{2}-y\right)^{2}=d_{2}^{n} \\
\cdots \cdots \\
\left(x_{n}-x\right)^{2}+\left(y_{n}-y\right)^{2}=d_{n}^{2}
\end{array}\right.
$$

Given equation set (3) is non-linear and hence difficult to solve, so we rewrite it for solution [9]:

$$
A X=B
$$

Where, $A=\left[\begin{array}{cc}2\left(x_{1}-x_{n}\right) & 2\left(y_{1}-y_{n}\right) \\ 2\left(x_{2}-x_{n}\right) & 2\left(y_{2}-y_{n}\right) \\ \ldots & \ldots \\ 2\left(x_{n-1}-x_{n}\right) & 2\left(y_{n-1}-y_{n}\right)\end{array}\right], B=\left[\begin{array}{c}x_{1}^{2}-x_{n}^{2}+y_{1}^{2}-y_{n}^{2}+d_{n}^{2}-d_{1}^{2} \\ x_{2}^{2}-x_{n}^{2}+y_{2}^{2}-y_{n}^{2}+d_{n}^{2}-d_{2}^{2} \\ \ldots \ldots \\ x_{n-1}^{2}-x_{n}^{2}+y_{n-1}^{2}-y_{n}^{2}+d_{n}^{2}-d_{n-1}^{2}\end{array}\right]$, and $X=(x, y)^{T}$.

Least square method is used to solve the equation above, we obtain: $X=\left(A^{T} A\right)^{-1} A^{T} B$.

\section{Weighted Centroiding Positioning}

The basic idea of centroiding algorithm is to obtain the information of reference nodes within the communication range of unknown node, and use the geometric centroid of these reference nodes as the its position [10]. Compared with other positioning algorithms, centroiding positioning algorithm is relatively simple, so it needs no extra hardware support. However, the accuracy of this algorithm is closely related to the density of reference nodes. 


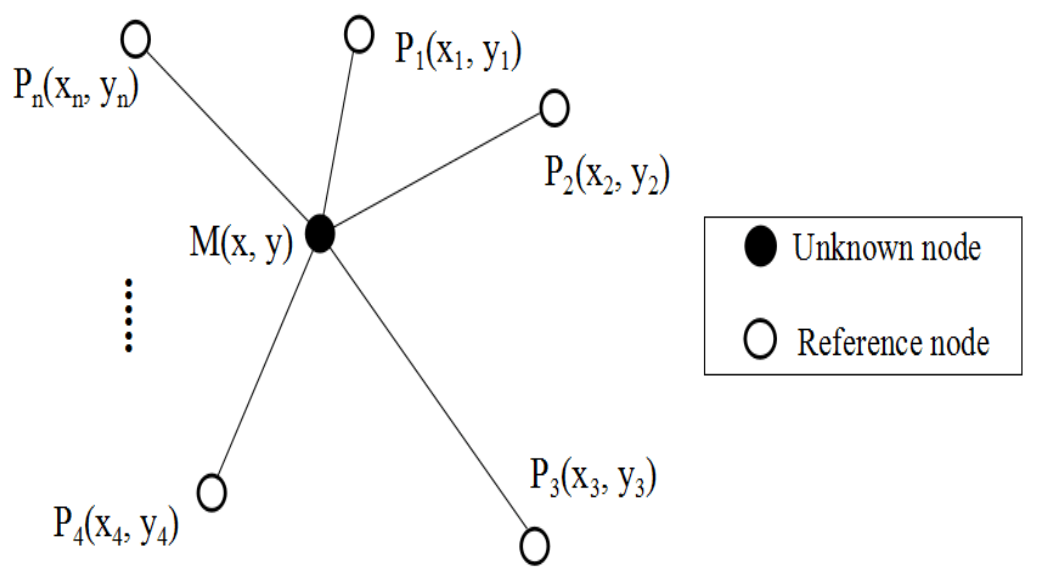

Fig.1. Centroiding Positioning Algorithm

As shown in Fig.1, considering the coordinate of unknown node is $M(x, y)$, the coordinates of $n$ reference nodes are $P_{1}\left(x_{1}, y_{1}\right), P_{2}\left(x_{2}, y_{2}\right), P_{3}\left(x_{3}, y_{3}\right), \ldots, P_{n}\left(x_{n}, y_{n}\right)$, following formula is provided for position:

$$
(x, y)=\left(\frac{x_{1}+x_{2}+\ldots+x_{n}}{n}, \frac{y_{1}+y_{2}+\ldots+y_{n}}{n}\right)
$$

The centroiding algorithm above does not reflect the different influences of reference nodes, so weighted centroiding algorithm [11] is invented, in which weight factor is added to evaluate different reference nodes. Various types of weight factors can be adopted. For example, the RSSI-based weighted centroiding algorithm [12] uses RSSI value of reference node as the corresponding weight.

\section{Proposed Hybrid Algorithm}

This proposed hybrid algorithm mainly includes two stages. In the first stage, rough estimation on the unknown node according to the maximum likelihood estimation method. In the second stage, and weighted centroiding algorithm is used to improve the positioning accuracy.

In order to take into consideration the different locations of reference nodes, a new type of weight factor, namely square difference, is introduced in. Take the horizontal coordinates as an example. Assuming the average value of horizontal coordinates of $\mathrm{n}$ known nodes is $x_{0}$, the weight is defined as $w_{i x}=x_{i}^{2}-x_{0}^{2}$, where different locations corresponds with different weight values. Similarly, for vertical coordinates, the weight is $w_{i y}=y_{i}^{2}-y_{0}^{2}$. Then formula after modification runs as follows:

$$
(x, y)=\left(\frac{\sum_{i=1}^{n}\left(w_{i x} x_{i}\right)}{\sum_{i=1}^{n} w_{i x}}, \frac{\sum_{i=1}^{n}\left(w_{i y} y_{i}\right)}{\sum_{i=1}^{n} w_{i y}}\right)
$$

The detailed steps of our hybrid algorithm are listed as follows:

(1) record the RSSI of $m$ reference nodes, and calculates the distances with path loss model. If more than $m$ reference nodes are detected, choose $m$ reference nodes with higher RSSI.

(2) arbitrary select $m-2$ reference nodes to apply maximum likelihood estimation and get $C_{m}^{2}=\frac{m(m-1)}{2}=n$ rough estimated results.

(3) apply weighted centroiding algorithm to handle $n$ estimated results, with the weight factor $w_{i x}=x_{i}^{2}-x_{0}^{2}$ and $w_{i y}=y_{i}^{2}-y_{0}^{2}$, where $x_{0}=\frac{x_{1}+x_{2}+\ldots+x_{n}}{n}$ and $y_{0}=\frac{y_{1}+y_{2}+\ldots+y_{n}}{n}$. 


\section{Test Results Analysis and Comparison}

A passive Wireless Fidelity (WiFi) positioning system is built up to examine the performance of proposed hybrid algorithm, as shown in Fig.2. In this system, the position target (unknown node) is a mobile phone with WiFi interface, and the reference nodes are some WiFi Access Points (APs). The RSSI between mobile phone and AP is continuously measured by AP, sent to a positioning server via Socket and stored in a positioning database. When the mobile phone needs to be tracked, the positioning engine reads the RSSI values from database, and calculates its coordinate according to the proposed hybrid algorithm and other algorithms.

The test is conducted in a lab with the size of $10.8 \mathrm{~m} * 7.2 \mathrm{~m}$. One corner of the room is regarded as the original point, with the two sides of the corner being $\mathrm{X}$ and $\mathrm{Y}$ axles (unit: $\mathrm{m}$ ). Seven APs and one mobile phone are placed in the room. The coordinates of the seven APs are respectively AP1(3.6, 1.8), AP2(1.8, 0.6), AP3(5.4, 6.0), AP4(6.0, 7.8), AP5(2.4, 6.0), AP6(0, 4.2), AP7(7.2, 2.4). The actual position of the mobile phone is $(3.6,3.6)$.

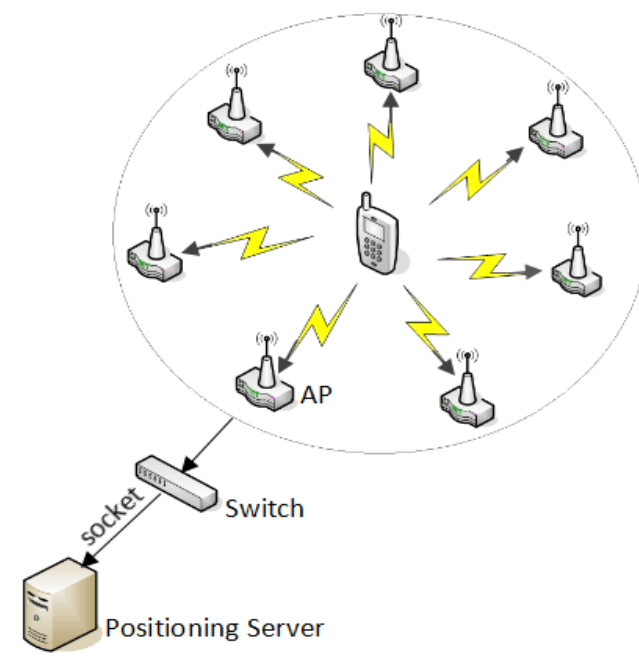

Fig.2. Passive WiFi Positioning System.

After starting the positioning system, the RSSI measured by each AP is automatically stored in the database. Part of RSSI data is shown as follows:

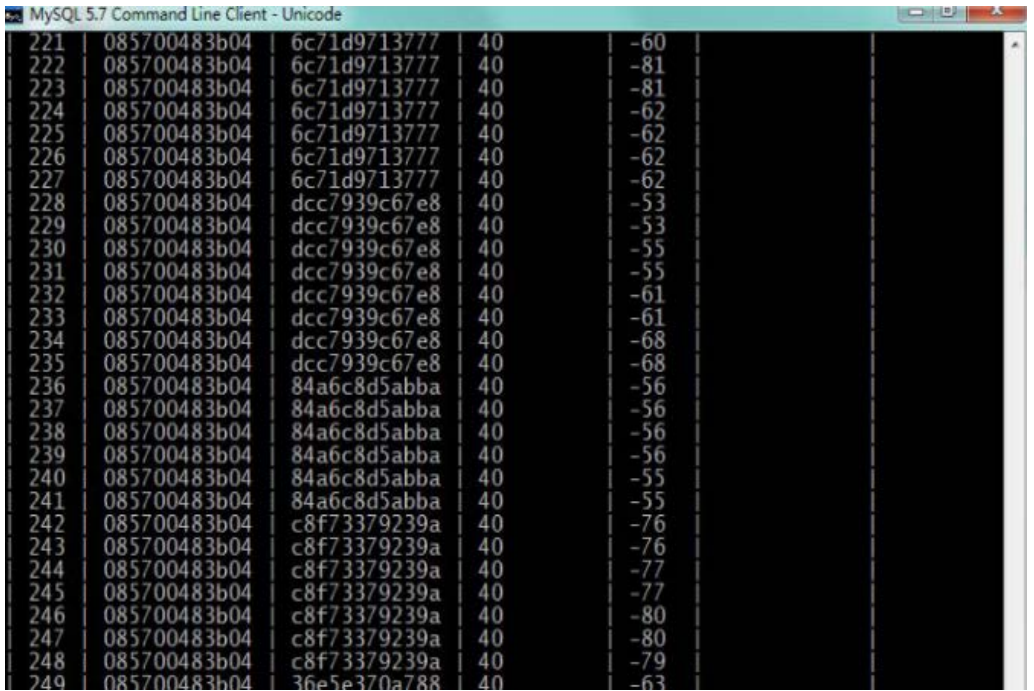

Fig.3. Positioning database records

Five columns of the database are record number, mac address of mobile phone, mac address of AP, Packet type and RSSI value, respectively. RSSI data from the same AP is averaged, producing the results $-60.90,-68.25,-68.00,-64.80,-67.71,-65.70$ and -68.50 , respectively. Fig.4 presents 21 rough positioning results of seven reference nodes by maximum likelihood estimation. Fig.5 compares the positioning results of hybrid positioning algorithm and two other traditional algorithms. Table 1 lists the absolute positioning errors of three algorithms. 


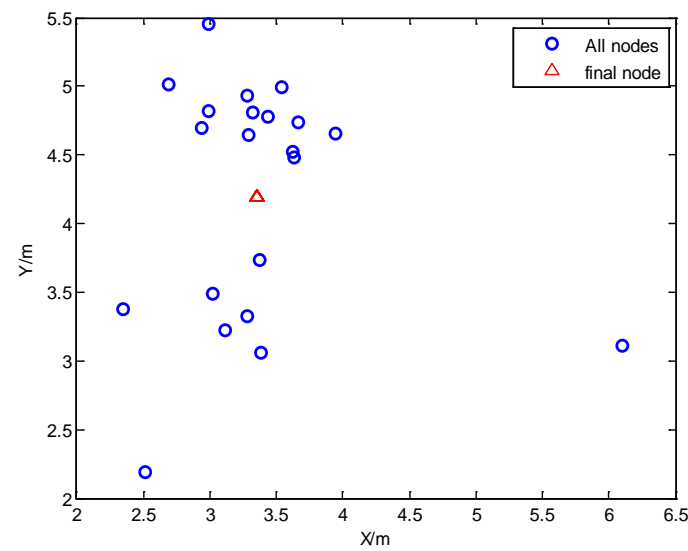

Fig.4. Roughly Estimated Points

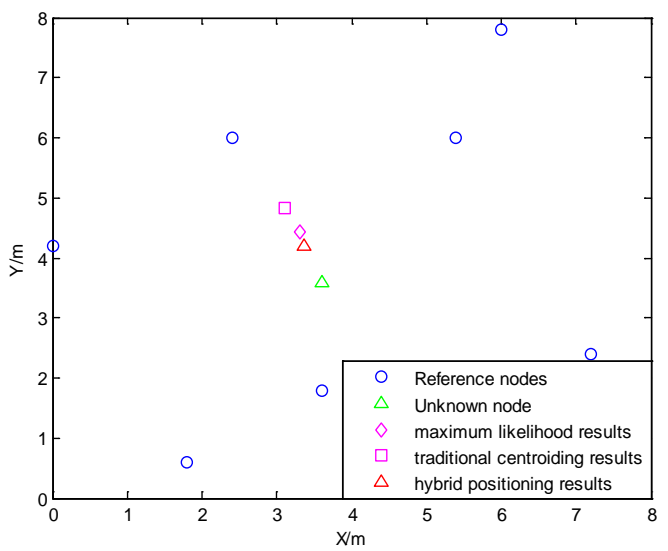

Fig.5. Comparison of three algorithms

Table 1 Comparison about the Positioning Accuracy of the Three Algorithms

\begin{tabular}{ccc}
\hline Positioning Algorithm & Positioning Results & Positioning Accuracy (absolute error) \\
\hline Maximum likelihood & $(3.3045,4.4316)$ & 0.8825 \\
Traditional centroiding & $(3.1023,4.8316)$ & 1.3284 \\
Proposed hybrid & $(3.3554,4.1919)$ & 0.6406 \\
\hline
\end{tabular}

Simulation results show that the hybrid algorithm proposed in this paper causes less positioning deviation compared with traditional centroiding algorithm and maximum likelihood algorithm, hence obtains a better positioning accuracy.

\section{Conclusions}

A hybrid positioning algorithm has been proposed in this paper by combining RSSI-based maximum likelihood estimation algorithm and weighted centroiding algorithm. It firstly roughly estimates the position information of positioning target, and then uses weighted centroiding algorithm to further improve the positioning accuracy. The proposed algorithm has been tested on our passive WiFi positioning system. Test results verify the effectiveness of the proposed algorithm.

\section{Acknowledgement}

This research is supported by Huaqiao University (No.12BS219, 13BS101).

\section{References}

[1] Li Mingfeng. GPS Positioning Technology and Its Application [M]. Beijing: National Defence Industry Press, 2006.

[2] Wang Bo, Xu Yubin. Range-free Positioning Algorithm of Nodes in Wireless Sensor Network[J]. Journal of Taiyuan University of Science and Technology, 2009, 30(6):466-471.

[3] Li Jianzhong, Gao Hong. Research Progress of Wireless Sensor Network[J]. Computer Research and Development, 2008, 45(1):1-15.

[4] Luthy, K. A., E. Grant, and T. C. Henderson. Leveraging RSSI for Robotic Repair of Disconnected Wireless Sensor Networks. IEEE International Conference on Robotics \& Automation 2007:3659-3664.

[5] Wu Bin, Li Jun'e. Application Research on Wireless Sensor Network in Indoor Positioning[J]. Computer Science, 2013(05):115-117.

[6] Chen Weike, Li Wenfeng, Shou Xing, et al. RSSI-based Weighted Centroiding Positioning 
Algorithm of Wireless Sensor Network[J]. Journal of Wuhan University of Technology (Communication Sciences and Engineering Edition), 2006, 30(2):265-268.

[7] Zhong Lihong, Hu Chengquan, Jin Jingji. Analysis and Realization of RSSI-based Maximum Likelihood Estimation Positioning Algorithm[J]. Journal of Jilin University (Natural Sciences Edition), 2014(3):556-560.

[8] Li Gang, Chen Junjie. WSN Three-dimensional Positioning Based on Beacon Node and RSSI Self-correcting[J]. Journal of Huazhong University of Science and Technology (Natural Sciences Edition), 2011,39(2):347-350

[9] Chen, Demin, and Y. Zhang. Research of WSN Localization Algorithm Based on Entropy Function. First International Workshop on Education Technology and Computer Science IEEE Computer Society, 2009:229-233.

[10] Deng Binwei, Huang Guangming. Positioning Algorithm of Wireless Sensor Network Supported by Mobile Nodes[J]. Chinese Journal of Scientific Instrument, 2011, 32(3):563-570.

[11] Zhu Bo, Chen Shu. On a Modified Algorithm of Centroiding Positioning in Wireless Sensor Network[J]. Journal of Sensor Technology, 2010, 23(6):868-872.

[12] Fu Hua, Hu Yaxin. RSSI-based Centroiding Positioning Algorithm in Wireless Sensor Network[J]. Microcomputer Information, 2010, 26(22):19-20. 